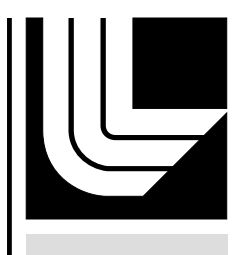

LAW RENCE LIVERMORE N A T IO N A L LABORATORY

\title{
Environmentally Benign Stab Detonators
}

A.E. Gash

January 6, 2005 
This document was prepared as an account of work sponsored by an agency of the United States Government. Neither the United States Government nor the University of California nor any of their employees, makes any warranty, express or implied, or assumes any legal liability or responsibility for the accuracy, completeness, or usefulness of any information, apparatus, product, or process disclosed, or represents that its use would not infringe privately owned rights. Reference herein to any specific commercial product, process, or service by trade name, trademark, manufacturer, or otherwise, does not necessarily constitute or imply its endorsement, recommendation, or favoring by the United States Government or the University of California. The views and opinions of authors expressed herein do not necessarily state or reflect those of the United States Government or the University of California, and shall not be used for advertising or product endorsement purposes.

This work was performed under the auspices of the U.S. Department of Energy by University of California, Lawrence Livermore National Laboratory under Contract W-7405-Eng-48. 


\section{Environmentally Benign Stab Detonators}

$$
\text { PP-1362 }
$$

\section{Lawrence Livermore National Laboratory}
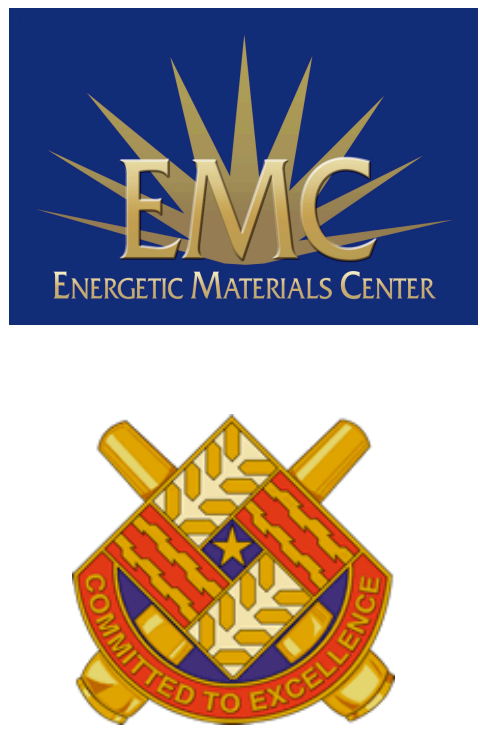

PI: Dr. Alexander Gash

P.O. Box 808 L-092

Livermore, CA 94551

925-423-8618

gash2@llnl.gov

Contributors:

Dr. Troy Barbee (LLNL)

Mr. Gartung Cheng (ARDEC, Picatinny)

Mrs. Neha Mehta (ARDEC, Picatinny) 


\section{Contents}

Page

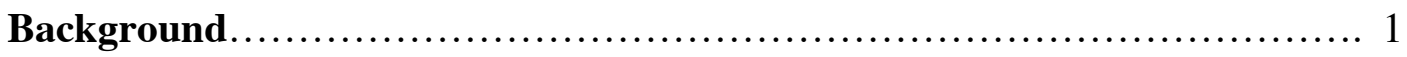

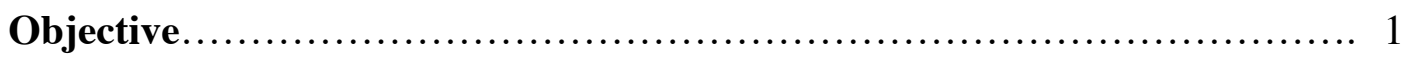

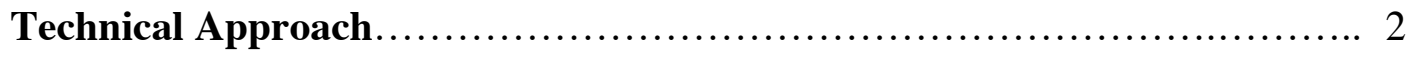

Nanolaminate materials and technology.............................. 2

Sol-gel energetic materials ........................................... 4

Integration of the nanotechnologies..................................... 5

Interaction with Army and transition plans........................... 5

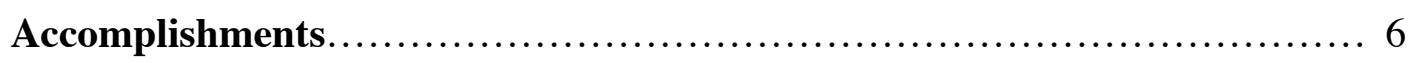

Demonstration that reacting laminate will initiate lead azide............... 6

Fabrication of nanolaminate thin films with varied structures .............. 7

Sensitivity of nanolaminate to mechanical ignition can be engineered ...... 11

Candidate materials screened for stab sensitivity at LLNL................. 13

Application based testing at ARDEC-TACOM........................... 15

Attempts to physically sensitize the nanolaminates. ........................ 19

New Sol-gel synthesis of nanometric tungsten (VI) oxide $\left(\mathrm{WO}_{3}\right) \ldots \ldots \ldots \ldots .21$

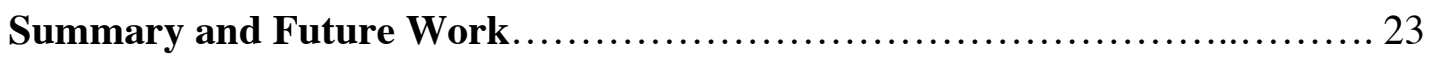

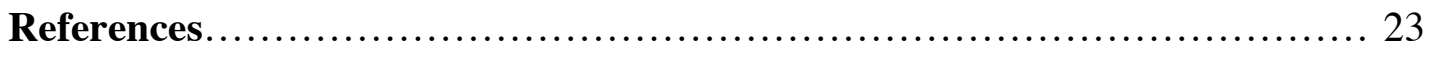

\section{List of Figures and Tables}

Figure 1. TEM image of multilayer fabricated at LLNL...................... 3

Figure 2. Photo summary of initiation of lead azide with reacting nanolaminate.. 6

Figure 3. Schematic representation of sol-gel coated nanolaminate ............. 9

Figure 4. DSC scans of candidate nanolaminate materials...................... 10 
Figure 5. Photo of a nanolaminate ignited by impact initiation................. 14

Figure 6. Photos of both un-reacted and reacted nanolaminate................. 15

Figure 7. Photo of a punched discs of $\mathrm{Al} /$ monel 400 nanolaminate.............. 16

Figure 8. Photo of M55 detonator cup, loaded with nanolaminate............... 16

Figure 9. Schematic of current M55 detonator and components................. 17

Figure 10. Drop-weight test apparatus at ARDEC-TACOM................... 18

Figure 11. Photo of pierced M55 detonator cup............................. 19

Figure 12. Photo of M55 stab detonator pin............................. 20

Figure 13. Mechanical ignition of sol-gel coated nanolaminate................ 20

Figure 14. Sol-gel derived $\mathrm{WO}_{3}$ gel.................................... 22

Figure 15. Spin coater and nanolaminate spun coated with sol-gel energetic..... 22

Table 1. Summary of structure of energetic nanolaminates made at LLNL......... 8

Table 2. Summary of structural parameters of nanolaminate foils engineered for enhanced sensitivity. ........................................................ 8

Table 3. Summary of results of small-scale safety testing for nanolaminates..... 12

Table 4. Results of LLNL drop testing of nanolaminate materials.............. 14 


\section{Background}

Many energetic systems can be activated via mechanical means. Percussion primers in small caliber ammunition and stab detonators used in medium caliber ammunition are just two examples. Current medium caliber $(20-60 \mathrm{~mm})$ munitions are detonated through the use of impact sensitive stab detonators. Stab detonators are very sensitive and must be small, as to meet weight and size limitations. A mix of energetic powders, sensitive to mechanical stimulus, is typically used to ignite such devices. Stab detonators are mechanically activated by forcing a firing pin through the closure disc of the device and into the stab initiating mix. Rapid heating caused by mechanically driven compression and friction of the mixture results in its ignition. The rapid decomposition of these materials generates a pressure/temperature pulse that is sufficient to initiate a transfer charge, which has enough output energy to detonate the main charge. This general type of ignition mix is used in a large variety of primers, igniters, and detonators.[1]

Common primer mixes, such as NOL-130, are made up of lead styphnate (basic) $40 \%$, lead azide (dextrinated) $20 \%$, barium nitrate $20 \%$, antimony sulfide $15 \%$, and tetrazene 5\%.[1] These materials pose acute and chronic toxicity hazards during mixing of the composition and later in the item life cycle after the item has been field functioned. There is an established need to replace these mixes on toxicity, health, and environmental hazard grounds.

\section{Objective}

This effort attempts to demonstrate that environmentally acceptable energetic solgel coated flash metal multilayer nanocomposites can be used to replace current impact initiated devices (IIDs), which have hazardous and toxic components. Successful completion of this project will result in IIDs that include innocuous compounds, have sufficient output energy for initiation, meet current military specifications, are small, cost competitive, and perform as well as or better than current devices. We expect flash metal multilayer and sol-gel to be generic technologies applicable to a wide range of devices, especially in small caliber ammunition and sub-munitions.

We will replace the NOL-130 mixture with a nanocomposite that consists of a mechanically robust energetic multilayer foil that has been coated with a sol-gel energetic material. The exothermic reactions are activated in this nanocomposite are the transformation of the multilayer material to its respective intermetallic alloy and the thermite reaction, which is characterized by very high temperatures, a small pressure pulse, and hot particle ejection. The proposed materials and their reaction products consist of, but are not limited to aluminum, nickel, iron, aluminum oxide, titanium, iron 
oxide and boron. These materials have much more desirable environmental and health characteristics than the NOL-130 composition.

\section{Technical Approach}

Our objective is being accomplished through the development of a device(s) that consists of an energetic nanometer metallic multilayer coated with a sol-gel derived energetic nanocomposite. The proposed IIDs will be made up of a precision energetic foil of metal multilayers (A.K.A. flash metal) along with a ceramic-based energetic solgel coating made up of non-toxic and non-hazardous components such as ferric oxide and aluminum metal. The multilayer foils can be produced using magnetron physical vapor sputtering techniques. Both the multilayer and sol-gel technologies are versatile commercially viable processes that allow the "tailoring" of properties such as stab sensitivity and energy output. In this work, the flash metal serves as the precision igniter and the energetic sol-gel functions as a low-cost, non-toxic, non-hazardous booster in the ignition train. In contrast to other energetic nanotechnologies (i.e. mixing of nanometer powders (MIC)), these materials are safe, low cost, structurally robust, reproducible, and have excellent aging properties.

Our main interest in the sol-gel and multilayer sputtering techniques approach to energetic materials is that it offers the possibility to precisely control the composition and morphology of the target material at the nanometer scale which are important variables for both safety and reaction rates; a result that is difficult if not impossible to achieve by most conventional techniques. Such control of the nanostructure could enable the creation of entirely new energetic materials with desirable properties.

Nanolaminate materials and technology. Nanolaminates are metallic foils that are periodic in one dimension in composition or in composition and structure. They are made by alternate deposition of two or more materials. Variation is generated during the synthesis of the material, which is done atom by atom. Individual layers can be varied in

thickness from one atomic layer $(\sim 2 \AA)$ to thousands of atoms thick $(>10,000 \AA)$. Using this technology multilayer structure can be formed with microstructures and compositions that are not possible using traditional processing technology.

Multilayer structured materials can be formed by several different techniques. Physical vapor deposition, chemical vapor deposition, electrochemical deposition, electrolytic deposition, and atomic layer epitaxy are all utilized to prepare multilayer materials. One type of physical vapor deposition involves sputtering. In sputter deposition systems atoms, or clusters of atoms, are generated in the vapor phase by bombardment of a solid source material with energetic particles. The substrate is moved past the source(s) and vapor condenses on the substrate to form a film. A single layer of material is deposited on the substrate with each pass. The thickness of component layers, 
and thus it's resulting physical properties, is precisely controlled by adjusting the periodicity of substrate movement. Magnetron sputtering is one type of sputtering technique and it is the physical vapor method of choice for the semiconductor industry.

Over the last two decades researchers at LLNL have developed technology to deposit layers of atoms onto a substrate using magnetron-sputtering techniques. Alternating layers of different elements, each several nanometers thick, can be deposited on top of one another to make nanometer metallic multilayers with a thin intermixed region between the layers. The sum of the thicknesses of two adjacent layers in the structure is referred to as the bi-layer thickness and is an important structural and performance parameter of the material. Figure 1 is a transmission electron micrograph of a multilayer structure with the bi-layer thickness outlined.

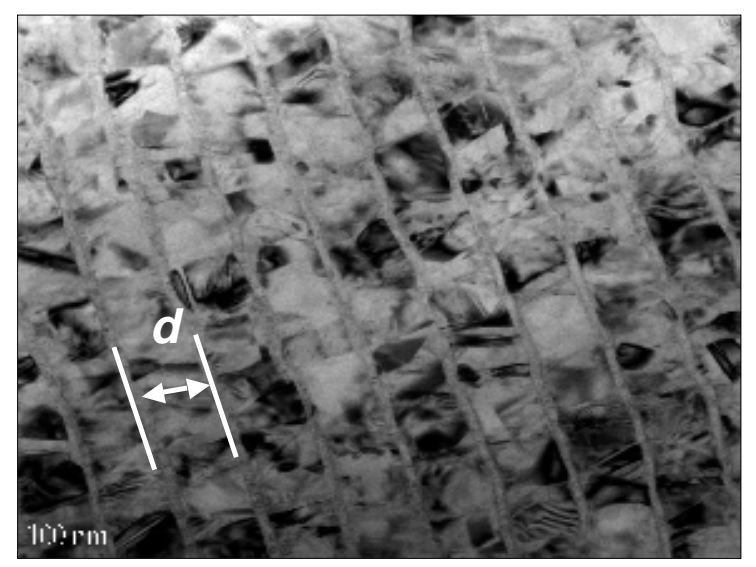

Figure 1. TEM image of a $\mathrm{Zr} / \mathrm{Cu}$ nanolaminate thin film, the bi-layer thickness value is shown by the distance $d$.

One additional structural feature of energetic multilayer thin films not well illustrated by the image in Figure 1 is the so-called pre-reaction zone. This region exists at the interface of adjacent layers of the multilayer and is made up of a thin layer of intermetallic product formed during deposition. The thickness of this region, relative to the overall period, is very important to the overall energy and sensitivity of the energetic nanolaminate. There is always a pre-reaction zone in metallic multilayer structures however its thickness can be controlled in many cases.

The properties of the multilayers are very dependent on structure and composition, both of which can be conveniently controlled by changing reactor conditions. Certain multilayers can be engineered to be energetic: The energy being derived from the rearrangement of some heterometallic multilayers into a more stable intermetallic compound. These flash metals have been shown to be sensitive to both thermal and mechanical stimuli.[2,3] The stored energy and reaction velocities of the 
multilayers can be systematically and independently controlled by materials selection and size scale of the layers. In many cases the flash metals have the structural properties of a robust foil. This technique is very versatile as nearly all metals can be utilized to make tailored energetic multilayers and thus compositional control is vast. As the method is extremely reproducible, it is anticipated that the precision of the IIDs will be very high. Furthermore, the metallic multilayers have been shown to have good environmental stability as they are currently used as precision reflective coatings on orbiting satellites such as the Transition Region and Corona Explorer and are thus exposed to harsh environments and stresses. This is a major issue that may prove to be an insurmountable obstacle to approaches using nanometer metal fuel powders (i.e., MIC materials) in the initiating mix. In addition, the method to prepare flash metals is affordable and the capital equipment and infrastructure currently exists in industry to do so.

Multilayer flash metal materials can be prepared with tailored and precise reaction wave front velocities, energy release rates, and ignition temperatures. For example, the velocity of a multilayer thin film depends on the relative thickness and composition of each multilayer structure. Reaction front velocities from 0.2-100 meters/second can be prepared reliably and precisely. Multilayer reaction temperatures between 200 and 1500 ${ }^{\circ} \mathrm{C}$ are observed for multilayers with different compositional and structural characteristics. Heats of reaction from 0.1-1.8 $\mathrm{kcal} / \mathrm{g}$ are capable with different multilayers.[2-5] There have been several reports on the modeling and characterization of these properties and the influence of structure, composition, and processing conditions on such variables.

Sol-gel energetic materials. Sol-gel chemical methodology has been investigated for approximately 150 years and has been extensively employed in the disciplines of chemistry, materials science, and physics. In fact, there are few scientific fields that have not benefited, in some fashion, from the sol-gel method and its various applications. Solgel chemistry is a solution phase synthetic route to highly pure organic or inorganic materials that have homogeneous particle and pore sizes as well as densities. Its benefits include the convenience of low-temperature preparation using general and inexpensive laboratory equipment. From a chemical point of view, the method affords easy control over the stoichiometry and homogeneity that conventional methods lack. One of the integral features of the method is its ability to produce materials with special shapes such as monoliths, fibers, films, coatings, and powders of uniform and very small particle sizes.

The $\mathrm{pH}$ of the solution, the solvent, the temperature, and the concentrations of reactants used can dictate the size of the clusters, which can be from $1 \mathrm{~nm}$ to $1000 \mathrm{~nm}$ in diameter. By controlling the conditions in solution, the sol can be condensed into a robust gel. The linking together of the sol clusters into either aggregates or linear chains results in the formation of the stiff monolith. The gel can be dried by evaporation of the solvent to produce a xerogel or removed under the supercritical conditions of the pore 
liquid to produce an aerogel. A typical gel structure is characteristically very uniform as the particles and the pores between them are nanometer-sized. This homogeneity leads to very uniform materials properties of sol-gel derived materials. It should be added that this is a bulk technique and in the case of atmospheric drying of xerogel coatings should require no special equipment and associated costs

Sol-gel techniques, developed at LLNL, provide a new approach to prepare energetic materials. LLNL was the first to appreciate that this methodology enables a unique way to the control of the morphology, size, and composition of components of energetic nanocomposites as well as enhancing their intimate mixing.[6-8] For the past fifteen years researchers at LLNL have developed a new economical, safe, and straightforward sol-gel synthetic routes to highly pure, high surface area, small particle size, inorganic oxides (oxidizers) and organic (fuel) sol-gel materials.[9-11] Using the sol-gel methodology structural and compositional parameters can be manipulated on the nanoscale. This has enabled the establishment of new energetic materials with new and potentially useful properties. With this method come new potential benefits of added safety, reproducibility, versatility, and low hazardous waste generation. LLNL has also developed a convenient and generic method for incorporating organic gas generating constituents into energetic ceramic/fuel metal thermite composites.

Integration of the two nanotechnologies. These two nanomaterials, nanolaminates and sol-gel materials, can be coupled to one another. One particularly attractive aspect of sol-gel methodology is that is very amenable to coating surfaces. Several mature methods such as spin-, dip-, or spray coating are currently used industrially to coat materials using sol-gel methods. The resulting materials can be relatively defect-free and durable, especially if the surface is smooth and clean. Fortunately sputtered multilayers have these characteristics. Our objective will be accomplished by developing a device(s) that consists of an energetic nanometer metallic multilayer coated with a sol-gel derived energetic nanocomposite.

Interaction with Army and transition plan. This is a joint effort between Lawrence Livermore National Lab (LLNL) and the U.S. Army's Armament Research Development and Engineering Center (ARDEC) at the Picatinny Arsenal, New Jersey. The partnership with ARDEC at Picatinny is very appropriate as they are the Army's leading research facility responsible for the manufacturing science and engineering of arsenal munitions and weapons and their production. Following successful completion of the Strategic Environmental Research and Development Program effort, the Energetics and Warhead Division of ARDEC at Picatinny has committed support for the transition of these materials to Army systems.

Initially we plan to focus on replacing the NOL-130 initiating mixture with a device that consists of a multilayer nanocomposite coated with a sol-gel energetic material. This material(s) will be prepared and physically characterized at LLNL and send to ARDEC where testing on its performance characteristics will be carried out. 
Tests will include those for impact energy sensitivity. Feed back from ARDEC will dictate the alteration of processing and compositional variables of the new IIDs at LLNL to alter the performance characteristics of the IID. Suitable candidate material(s) will be selected and ARDEC will attempt to couple these initiating mixtures to lead azide transfer charge. Witness plate testing will be done at ARDEC on these new stab detonators according to specifications outlined in MIL-D-50865, MIL-D-14978, or MILD-70436. Once the new initiating mixture has been shown to provide the necessary stimuli to transition the lead azide work will begin to find a suitable material to replace lead azide as the transition charge.

One promising candidate for the replacement of lead azide has already been identified in discussion with ARDEC. Upon anticipated selection of a suitable replacement for NOL-130, this device will be coupled with the candidate replacement charge material and the resulting IID will have its performance characterized. The lead azide in the transition charge needs to be replaced, however replacement of the NOL-130 initiating mixture alone would be an important development for other applications such as igniters and small caliber primers.

\section{Accomplishments}

Demonstration that reacting laminate will initiate lead azide. In the initial review of this proposed work some reviewers raised concerns as to whether the reacting nanolaminate had the necessary energetic output to initiate the lead azide transfer charge material. This was addressed by revising the proposal to include a decision point task, which one aspect was demonstrating the efficacy of reacting nanolaminate to initiate lead azide. Experiments to address this were performed at Picatinny. Aluminum/Monel 400 nanolaminates were used.
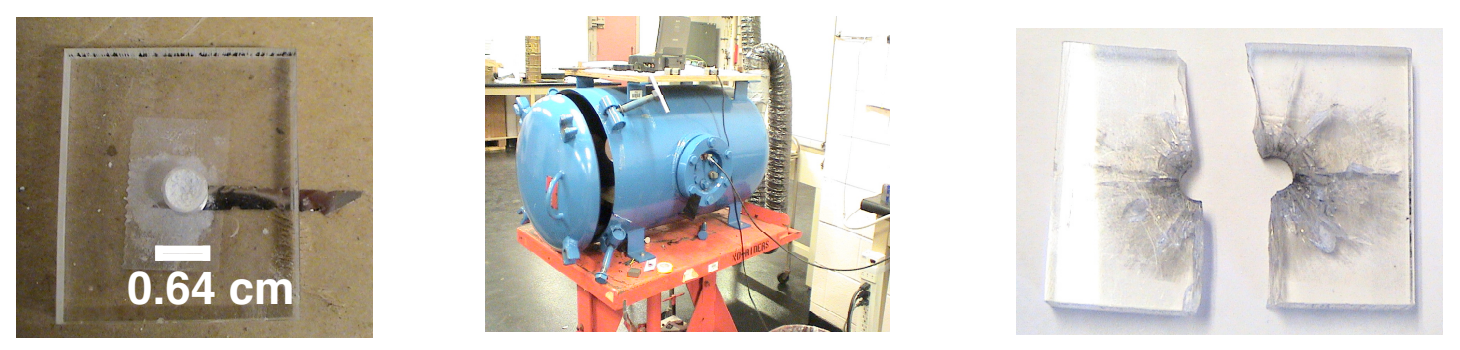

Figure 2. The photo on the top left is a Plexiglas plate with lead azide powder hand tamped in a hole in the middle. At the bottom of the plate is a nanolaminate foil strip that was in contact with the lead azide. The experiment test setup was put in a detonation chamber at Picatinny, shown above in the middle photo. The nanolaminate was ignited 
using a Nichrome hot wire. The reacting nanolaminate resulted in the detonation of the lead azide and shattering of the Plexiglas plate shown in the photo to the left.

The results of the experiment shown in Figure 2 are definitive: reacting nanolaminate will initiate the detonation of lead azide. A similar experiment was performed with the nanolaminate piece inserted into a M55 detonator cup with lead azide hand tamped on top. This also resulted in detonation of the lead azide. These experiments demonstrate that reacting nanolaminate will initiate the transfer charge material. Ideally this will be demonstrated, fully integrated, in a M55 detonator that is with transfer and output charge pressed and the cup sealed. Currently, efforts are being directed to demonstrate this. However this has proven difficult, as to date no nanolaminate material has been found to be stab sensitive at an input energy of less than $10 \mathrm{in./oz}$. This input energy value is important, as above it the transfer charge itself is stab sensitive. Thus, in any integrated experiment with an input energy above that level it will be impossible to determine if the nanolaminate has initiated the lead azide or the alternative. Nonetheless it has been demonstrated that the nanolaminate has sufficient energy output to initiate lead azide. The real issue is the sensitivity at this point of the project.

Similar experiments were also performed that demonstrate that the nanolaminate will also initiate the compound diaminohexazido cyclotetraphosphazene (DAHA). DAHA is a compound that is being considered as a non-toxic replacement for lead azide in the transfer charge of stab detonators. In addition, experiments are planned to determine if reacting nanolaminate can initiate another replacement charge candidate, ammonium bis(bi(tetralato)amineferrate(III), being developed at Los Alamos National Laboratory and tested at Picatinny.

Fabrication of nanolaminate thin films with varied structures. Of specific interest to this project are the structural parameters of the nanolaminate and how they affect sensitivity and energy output of the device. Qualitative reasoning suggests that by decreasing the thickness of individual layers in the nanolaminate the reaction speed and sensitivity of the material would increase. The rationale for this is with decreasing bilayer thickness (see Figure 1) the average diffusion distance between reactant species in adjacent layers decreases. A more detailed understanding of this reasoning is needed. As was stated in the previous section our central focus now is on enhancing the sensitivity of the foils to mechanical stimulus, it is the key factor for continued advancement of the project. The bi-layer thicknesses of the foils can be readily and precisely controlled via magnetron sputtering. Therefore a series of nanolaminates with varied structure and composition were prepared at LLNL for this project. A summary of the energetic nanolaminates fabricated at LLNL is given in Tables 1 and 2 below. 
Table 1. Summary of structure of energetic nanolaminates made at LLNL.

\begin{tabular}{|c|c|c|c|c|c|}
\hline \multirow[b]{2}{*}{ Sample } & \multicolumn{5}{|c|}{ Structure } \\
\hline & Composition & $\begin{array}{l}\text { \# Top } \\
\text { Layers }\end{array}$ & $\begin{array}{l}\# \mathrm{Al} / \mathrm{X} \\
\text { Layers }\end{array}$ & Thickness & $\begin{array}{c}\text { \# Bottom } \\
\text { Layers }\end{array}$ \\
\hline 294-TA21 & Al/Monel 400 & 10 & 1175 & $\begin{array}{c}20 \mathrm{~nm} \text { Al-13 nm } \\
\text { Monel }\end{array}$ & 10 \\
\hline 294-TA9 & Al/Monel 400 & 5 & 750 & $\begin{array}{c}36 \mathrm{~nm} \text { Al-12 nm } \\
\text { Monel }\end{array}$ & 5 \\
\hline 294-TA46 & Al/Monel 400 & 12 & 1432 & $\begin{array}{c}10 \mathrm{~nm} \mathrm{Al}-7 \mathrm{~nm} \\
\text { Monel }\end{array}$ & 12 \\
\hline 294-TA16 & $\mathrm{Al} / \mathrm{Ti}$ & 5 & 555 & NA & 5 \\
\hline 294-TA35 & $\mathrm{Zr} / 2 \mathrm{Al}$ & 2 & 1000 & $26 \mathrm{~nm} \mathrm{Al-18} \mathrm{nm}$ & 2 \\
\hline
\end{tabular}

Table 2. Summary of structural parameters of nanolaminate foils engineered for enhanced sensitivity.

\begin{tabular}{|c|c|c|c|c|c|}
\hline Material & \# Top layers & $\begin{array}{c}\text { \# Al/X } \\
\text { Layers }\end{array}$ & $\begin{array}{c}\text { Al layer } \\
\text { (nm) }\end{array}$ & $\mathbf{X}$ layer $(\mathbf{n m})$ & $\begin{array}{c}\text { \# bottom } \\
\text { layers }\end{array}$ \\
\hline $\mathbf{A l} /$ Monel & None & 1351 & 10.4 & 6.8 & $15 \mathrm{~nm} \mathrm{Cu}$ \\
\hline $\mathbf{A l} / \mathbf{N i}(\mathbf{V})$ & None & 1442 & 10.6 & 7.5 & None \\
\hline $\mathbf{A l} / \mathbf{N i}(\mathbf{V})$ & None & 1442 & 9.9 & 7 & None \\
\hline
\end{tabular}

A short discussion of important terminology for nanolaminate foils is now warranted and will help with full comprehension of this report. The total thickness of the foil sample is given by the distance set off by the larger set of brackets in the lower portion of Figure 3. The distance corresponding to individual component layer thickness is set off by the small brackets in Figure 3. Finally, the term referred to as the period, $d$, of the material is the distance of the repeating sub unit structure that makes up the foil. In For example, in Figure 3 the period is the sum of the thicknesses of one $\mathrm{Al}$ and one Ni layer as together they make up the repeating substructure. One additional structural feature of energetic multilayer thin films not well illustrated by the image in Figure 3 is the so-called prereaction zone. This region exists at the interface of adjacent layers of the multilayer and is made up of a thin layer of intermetallic product (NiAl in Figure 3) formed during deposition. The thickness of this region, relative to the overall period, is very important to the overall energy and sensitivity of the energetic nanolaminate. There is always a pre- 
reaction zone in metallic multilayer structures however its thickness can be controlled in many cases. These parameters are important as they directly relate to the sensitivity and total energy output of the synthesized foils.

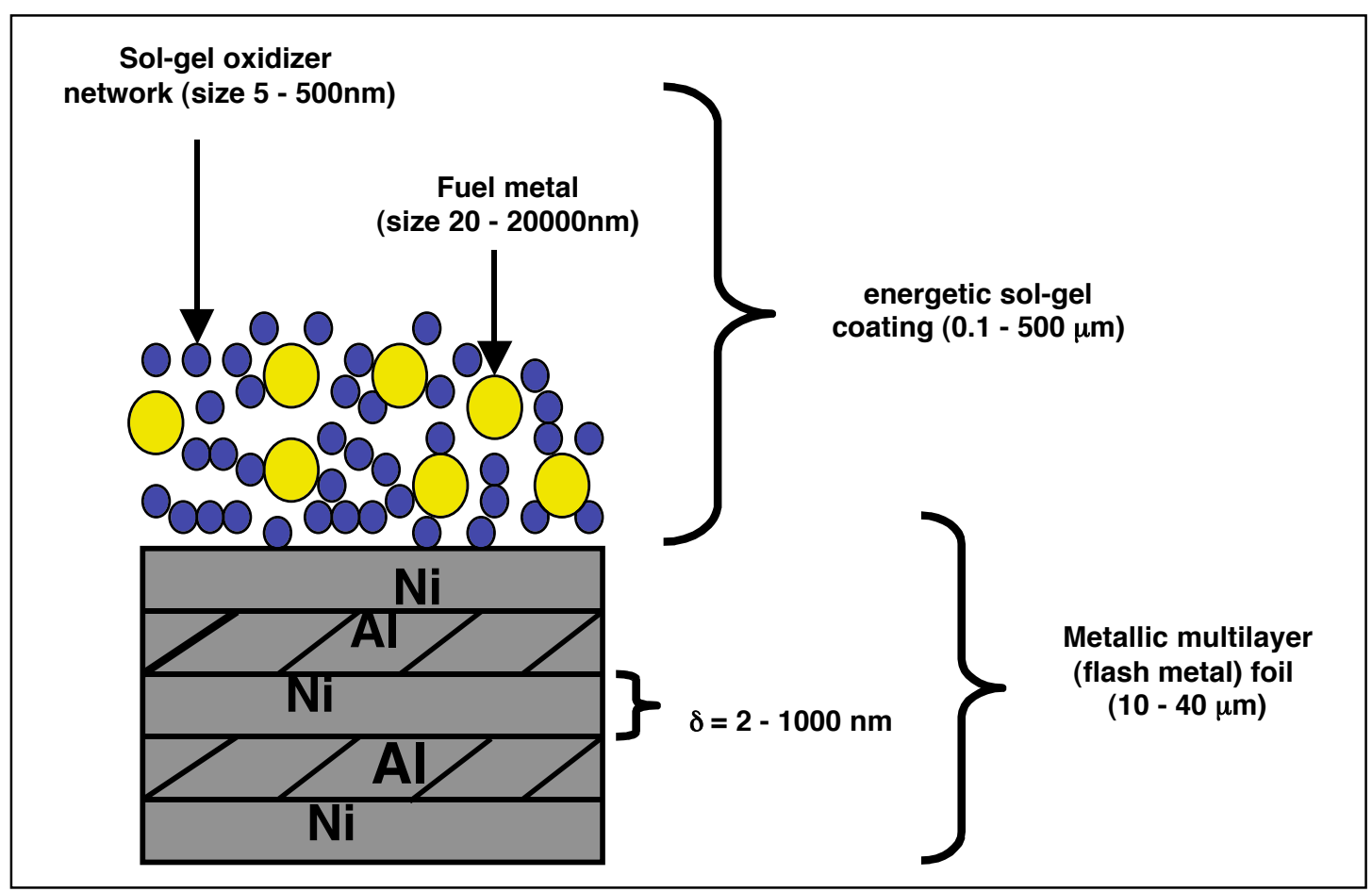

Figure 3. Scheme of microstructure of an energetic sol-gel coated flash metal multilayer nanocomposite for use as new, small, safe, and non-toxic initiators.

The materials summarized in Table 2 were prepared with the expressed goal of increasing the sensitivity to impact and friction stimulus and energy output. To achieve that, both structural and compositional parameters were varied. In all the cases in Table 2 the repeating bi-layer unit (see $d$ in Figure 1) was reduced relative to previous materials, see Table 1. Also one can see that the materials in Table 2 were not over-coated with a final finish of several metallic Al layers, as was done previously (see Table 1). With no protective overcoat these materials should be more sensitive to mechanical stimulus as less input energy will be utilized in deformation and mixing of adjacent layers with no heat of alloying. The materials summarized in Table 2 were fabricated with an emphasis to limit the thickness of the pre-reaction zone of adjacent layers. This was done using a specially designed water-cooled stage substrate for deposition that allowed the process to occur at $\sim 40^{\circ} \mathrm{C}$. It has been shown that a large pre-reaction zone has adverse effects on the total energy and reaction velocity of $\mathrm{Al} / \mathrm{Monel}$ multilayers.[2,3]. 
Compositionally the newer foils in Table 2 were prepared with components intended to have higher heats of reaction than those in Table 1. Differential scanning calorimetry (DSC) studies of the $\mathrm{Ni}(\mathrm{V}) / \mathrm{Al}$ multilayers show they are more energetic than the $\mathrm{Al} / \mathrm{Monel}$ ones. Figure 4 shows DSC traces for $\mathrm{Al} / \mathrm{Monel}$ and $\mathrm{Al} / \mathrm{Ni}(\mathrm{V})$ multilayers. As can be seen the integrated total energy values for the aluminum nickel system is approximately $15 \%$ greater than that for the Al/Monel system.[12-13] The nickel utilized in nanolaminates is stabilized with $7 \%$ vol. of vanadium. The vanadium is needed to stabilize the environment in the magnetron chamber and is essential.

a)

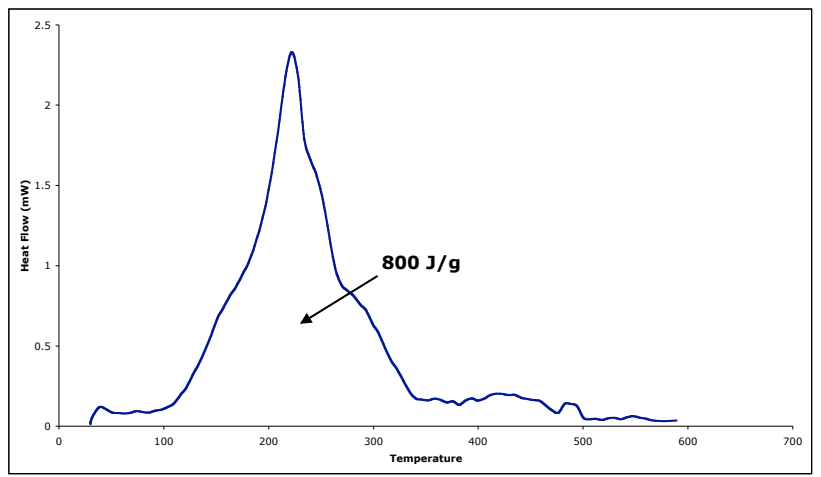

b)

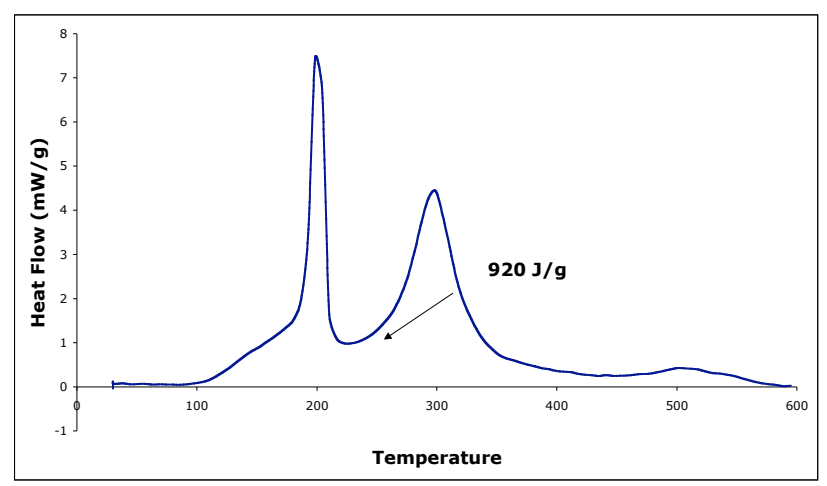

Figure 4a-b. a) Differential scanning calorimeter scan of Al/Monel 400 nanolaminate, b) DSC scan of $\mathrm{Al} / \mathrm{Ni}(\mathrm{V})$ nanolaminate.

This is a good point for a comment on the composition of materials shown in Tables 1 and 2, and its relevance to the goals for this project. Our main goal is to replace the toxic and hazardous components of current stab detonators with a more acceptable alternative in terms of performance and cost as well as health and environmental safety. 
Ideally this could be achieved with a benign replacement material. Reviewers of this project have expressed concern with the use of nickel in these replacement materials. Nickel has health and environmental concerns of its own and its inclusion in new materials may become problematic. Currently we are utilizing $\mathrm{Ni}$ or Ni-based alloy (Monel) in the many of the candidate nanolaminates. We feel this is justified and necessary. Historically we have the most experience with energetic nanolaminates that contain Ni. As this project is still in its basic proof of concept phase we felt it necessary to utilize materials that we have the most knowledge of and experience with. Once demonstrated successes have occurred we intend on utilizing a more acceptable material. We feel confident that a more acceptable material is possible as we have demonstrated energetic nanolaminate structures, with similar characteristics to those reported here, for multilayers containing aluminum and titanium or zirconium.

As was stated at the beginning of this section, the structural parameters engineered into these materials were done with the expressed goal of enhancing their sensitivity to impact and friction stimuli. Qualitative observations during handling of the materials in Table 2 indicate that this goal was achieved. We have noted that the materials appear to be more sensitive to ignition during handling processes such as cutting or punching of parts from the film. Some unintended ignitions have occurred, which we did not readily observe in handling the films in Table 1. Their enhanced sensitivity aside, films in Table 2 can be safely cut into smaller parts by following some guidelines we have established. Punching and shearing of the material with precision shim punches at room temperature appears to be very effective for safe handling. However, it is imperative that the punches and shears be kept very clean. We note that ignitions tend to occur much more frequently when a punch has been used several times without cleaning. Qualitatively these materials appear to be quite sensitive to lateral cutting action parallel to their layers that is typically introduced by a scoring or scraping actions. This could be due to the absence of over-coating layers, which may allow mixing of adjacent reactant layers with relatively little mechanical input. To address this development in more quantitative terms we performed small-scale safety testing on selected materials.

The sensitivity of nanolaminate foils to mechanical ignition can be engineered. Standard small-scale safety tests for explosives were performed on several energetic nanolaminates. These tests included the drop hammer test for impact sensitivity, the spark test for electrostatic sensitivity, and the B.A.M. friction test for friction sensitivity. [14] For reference the Drop Hammer at LLNL was calibrated with PETN, and RDX that have $\mathrm{DH}_{50}$ values of $15.5 \mathrm{~cm}$ and $34.5 \mathrm{~cm}$ respectively and the B.A.M. friction ignites PETN one out of 10 trials $(1 / 10)$ at $6.4 \mathrm{~kg}$. Most of these tests were performed at LLNL but some were performed at Picatinny, as noted in Table 3. 
Table 3. Results of small-scale safety testing of selected energetic nanolaminates

\begin{tabular}{|c|c|c|c|c|c|c|}
\hline Material & $\begin{array}{c}d \\
(\mathrm{~nm})\end{array}$ & $\begin{array}{c}\mathrm{Al} \\
(\mathrm{nm})\end{array}$ & $\begin{array}{l}\text { Metal } \\
(\mathrm{nm})\end{array}$ & Spark & $\begin{array}{l}\text { B.A.M. } \\
\text { Friction }\end{array}$ & $\begin{array}{c}\text { Drop } \\
\text { Hammer } \\
\left(\mathrm{DH}_{50}-\mathrm{cm}\right)\end{array}$ \\
\hline Al/Monel400 & 26.7 & 15.8 & 10.9 & NA & NA & $>100^{*}$ \\
\hline Al/Monel400 & 30.4 & 18 & 12.4 & $2 / 3 @ 0.04 J^{\star *}$ & $\begin{array}{c}1 / 10 \\
@ 10.8 \text { kg }\end{array}$ & 167.5 \\
\hline Al/Monel400 & 17.2 & 10.4 & 6.8 & 2/2@0.025J* & NA & $18^{*}$ \\
\hline $\mathrm{Al} / \mathrm{Ni}(\mathrm{V})$ & 13.6 & 7.95 & 5.65 & 2/2@ 0.04J** & $\begin{array}{c}1 / 10 @ 9 \\
4 \mathrm{~kg}\end{array}$ & 14.3 \\
\hline $\mathrm{Al} / \mathrm{Ni}(\mathrm{V})$ & 16.7 & 9.8 & 6.9 & $2 / 2 @ 0.04 J^{* *}$ & $\begin{array}{l}1 / 10 @ \\
3.4 \mathrm{~kg}\end{array}$ & 13.0 \\
\hline
\end{tabular}

*Tests performed at Picatinny

**This is the lowest setting for the spark testing apparatus at LLNL

There are several important conclusions that can be drawn from the data in Table 3. As a whole the nanolaminates with thinner layers of reactant materials and no overcoats are much more sensitive to impact at friction than those with thicker layers and capping layers of material (compare structural parameters of the last three materials in Table 3 to the first two). These are promising results as they indicate in a quantitative manner that the sensitivity of these energetic materials can be engineered. It is important to note that the influence of bi-layer thickness and over-coating layers does not appear to have the same drastic effect on spark sensitivity as it does on friction and impact sensitivity. Regardless of structure, all nanolaminates tested, via this method, here are very sensitive to spark discharge. In fact some appear to have the spark sensitivity level at the limit of primary explosives.

The implications of these testing results are very important for our continuing work. They demonstrate, quantitatively, that the sensitivity of nanolaminates to mechanical impact can be tailored and likely controlled through control of its microstructure. It has yet to be determined whether the root of this control is manifested through the bi-layer thickness, the pre-reaction thickness, or the extent of over-coating. 
Regardless it can be rationally controlled and provides strong encouragement for further experimentation to enhance sensitivity.

Candidate materials screened for stab sensitivity at LLNL. Although the smallscale safety tests reported in the previous section are encouraging it is important to understand that the replacement material(s) will need to be initiated by a stab input. There is not a great deal known about the mechanism(s) of stab initiation of energetic materials. The most definitive study, by Chaudhri, strongly suggests that the mechanism of stab initiation is largely frictional.[15] It is important to illustrate the difference between the mechanisms of initiation at work in the current system and those potential ones utilizing nanolaminates. In the current IIDs, the mechanical energy of a metal conical striker driving through a tightly packed powdered energetic material is degraded to heat that initiates the powder starting the detonation train. With nanolaminates there is no such powder. The "stab mix" is metallic foil disc(s) that are penetrated with a metal conical striker pin. There are significantly different mechanics between the two systems: the current powder-based one and the nanolaminates.

An important metric used in evaluating the efficacy of different materials as IIDs is impact energy. This can be envisioned as the amount of mechanical energy needed to cause the material to ignite/decompose. The firing energy for stab initiators is determined by a drop-weight test. In such a system a ball of a given weight is dropped from varying heights onto a centered firing pin that pierces the initiator mix. Many tests are run and the data is reduced to firing energy versus probability of initiation.[1] The sensitivity of IIDs to the drop ball test is reported in units of inch/ounces. That number can be thought of as how high a one-ounce ball needs to be dropped from to ignite the device. Most stab initiators function with high reliability between 0.5 and 5 in./oz. Percussion primers have higher firing energies, in the range of 18-60 in./oz.

ARDEC has a drop ball apparatus for measuring impact energies, as well as significant experience operating it and interpreting the results from testing. A modified drop-weight tests is used at LLNL for evaluating the qualitative stab sensitivity of nanolaminate films. In this test, nanolaminate foils are struck with a new detonator pin (M55 or M61) that has been hit with a one ounce steel ball dropped from varying heights. The nanolaminate rests on a small stainless steel anvil as it is struck. Using the LLNL drop-weight apparatus we have characterized the qualitative impact sensitivity of a series of nanolaminate materials. The results of our testing are shown below in Table 4. 
Table 4. Experimental results from sensitivity testing using a M55 detonator pin.

\begin{tabular}{|c|c|c|c|c|}
\hline Material & $\begin{array}{c}\text { Period } \\
\mathbf{( n m})\end{array}$ & $\begin{array}{c}\text { Al } \\
(\mathbf{n m})\end{array}$ & $\begin{array}{c}\mathbf{X} \\
\mathbf{( n m})\end{array}$ & $\begin{array}{c}\text { LLNL } \\
\text { Drop Test } \\
\text { (in./oz.) }\end{array}$ \\
\hline $\mathrm{Al} / \mathrm{Monel}$ & 33 & 19.9 & 13.1 & $>23^{*}$ \\
\hline $\mathrm{Al} / \mathrm{Monel}$ & 48 & 36 & 12 & $>23^{*}$ \\
\hline $\mathrm{Zr} / \mathrm{Al}$ & 44 & 26 & 18 & $>23^{*}$ \\
\hline $\mathrm{Zr} / \mathrm{Monel}$ & $\mathrm{NA}$ & $\mathrm{NA}$ & $\mathrm{NA}$ & $>23^{*}$ \\
\hline $\mathrm{Al} / \mathrm{Monel}$ & 17.2 & 10.4 & 6.8 & 12 \\
\hline $\mathrm{Al} / \mathrm{Ni}(\mathrm{V})$ & 16.9 & 9.9 & 7.0 & 15 \\
\hline $\mathrm{Al} / \mathrm{Ni}(\mathrm{V})$ & 15 & 8.8 & 6.2 & 15 \\
\hline
\end{tabular}

\section{* Limit of the LLNL instrument is a 23-inch drop.}

Table 4 summarizes the impact sensitivity of the nanolaminate foils along with pertinent structural and compositional factors for the films. Several materials showed no reaction to impact stimulus at the limit of our measurement (23 inches), however a few did show impact sensitivity. Figure 5 is a photo of a nanolaminate foil being ignited during a drop test. Two materials in particular have been identified as good candidates.

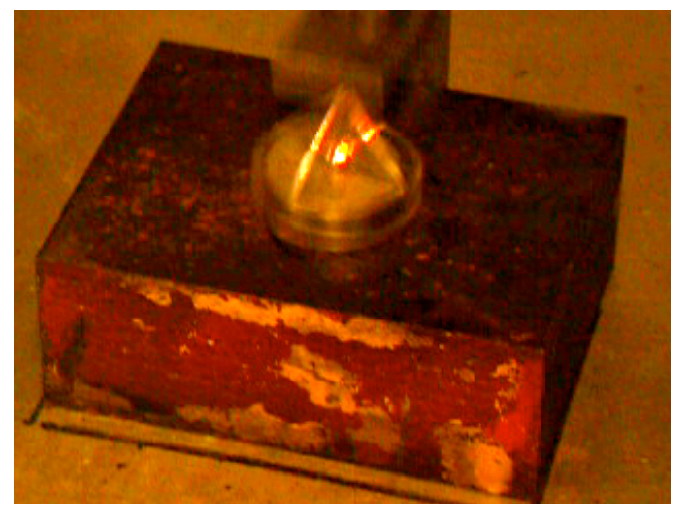

Figure 5. Photo of a nanolaminate ignited by impact initiation of a M55 stab pin on the LLNL drop test apparatus. ) 
One is an $\mathrm{Al} /$ monel 400 nanolaminate, the other a vanadium-doped nickel (7 vol.\% V)/Al nanolaminate. Monel 400 is an alloy of copper and nickel $\left(\mathrm{Cu}_{0.3} \mathrm{Ni}_{0.7}\right)$. We have been able to impact initiate foils of this material at energies as low as 12 in./oz. It is interesting to note that these materials were purposely engineered to have a very fine repeating bi-layer structure. Figure 6 contains photos of un-reacted and reacted foils from the LLNL drop testing.
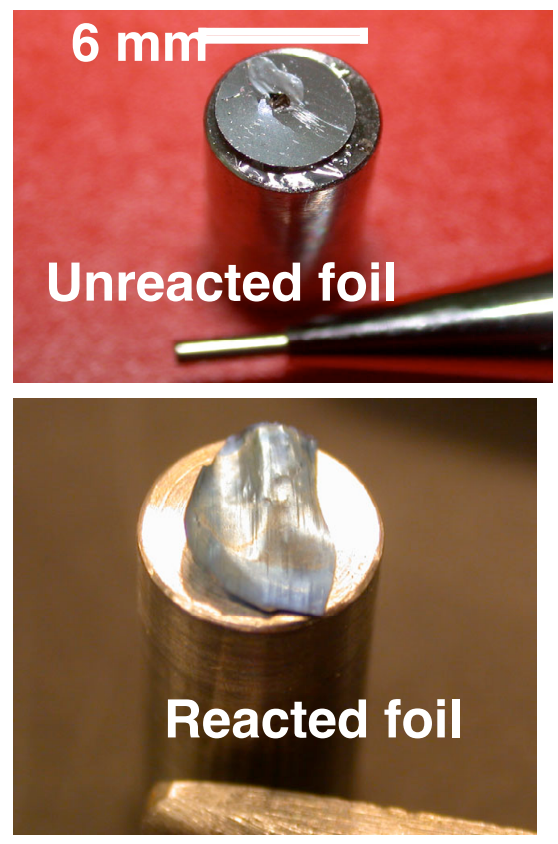

Figure 6. Photos of both un-reacted (top) and reacted (bottom) nanolaminate foils that have been initiated via mechanical impact.

The firing energy observed in the LLNL drop test for the nanolaminates is greater than that required for current stab detonators but less than that required for percussion primers. It is most desirable for new candidate IIDs to initiate at energies similar to current ones. We are currently engineering the sensitivity of the materials by varying the structural parameters of the nanolaminate as well as physical sensitization techniques.

Application based testing at ARDEC-TACOM. The successful demonstration of energetic nanolaminates with tailorable sensitivities led to further testing at ARDECTACOM in October 2004. Four materials, all of which showed enhanced sensitivity, 
were processed into several small diameter (3.2 and $4.8 \mathrm{~mm}$ diameter) disks, see Figure 7, and sent to the Army.

Materials were tested as follows: nanolaminate foil(s) discs from LLNL were inserted into the bottom of an aluminum M55 detonator cup. The remaining

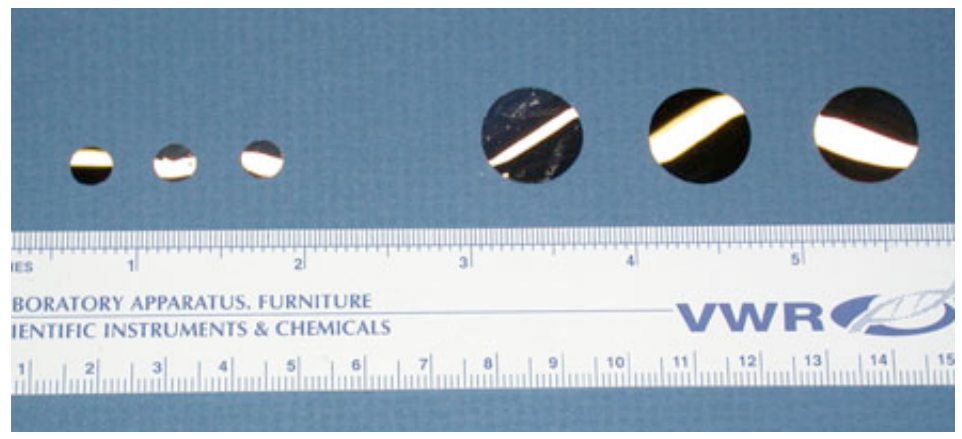

Figure 7. Photo of a punched discs of $\mathrm{Al} / \mathrm{monel} 400$ nanolaminate, small discs have a diameter of $0.64 \mathrm{~cm}$ and large discs are $1.60 \mathrm{~cm}$ in diameter.

volume of the cup was filled with a powder of the mineral talc (magnesium orthosilicate; Mohs hardness of 1.5) and the contents pressed to a pressure of 15,000 psi. Figure 8 is a photo of a nanolaminate-loaded M55 detonator.

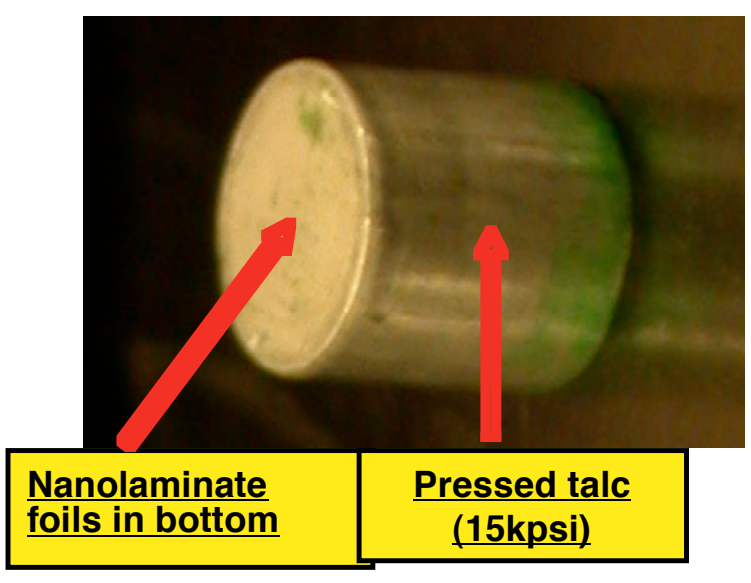

Figure 8. Photo of M55 detonator cup, the length of the cup is $4.8 \mathrm{~mm}$.

The talc is meant to be a surrogate material to mimic the mechanical properties of the transfer charge material in the stab detonator. In current stab detonators the transfer 
charge material is a powder that is pressed on top of the NOL-130 stab mix. Figure 9 shows the composition and arrangement of the detonation train components in a M55 stab detonator.

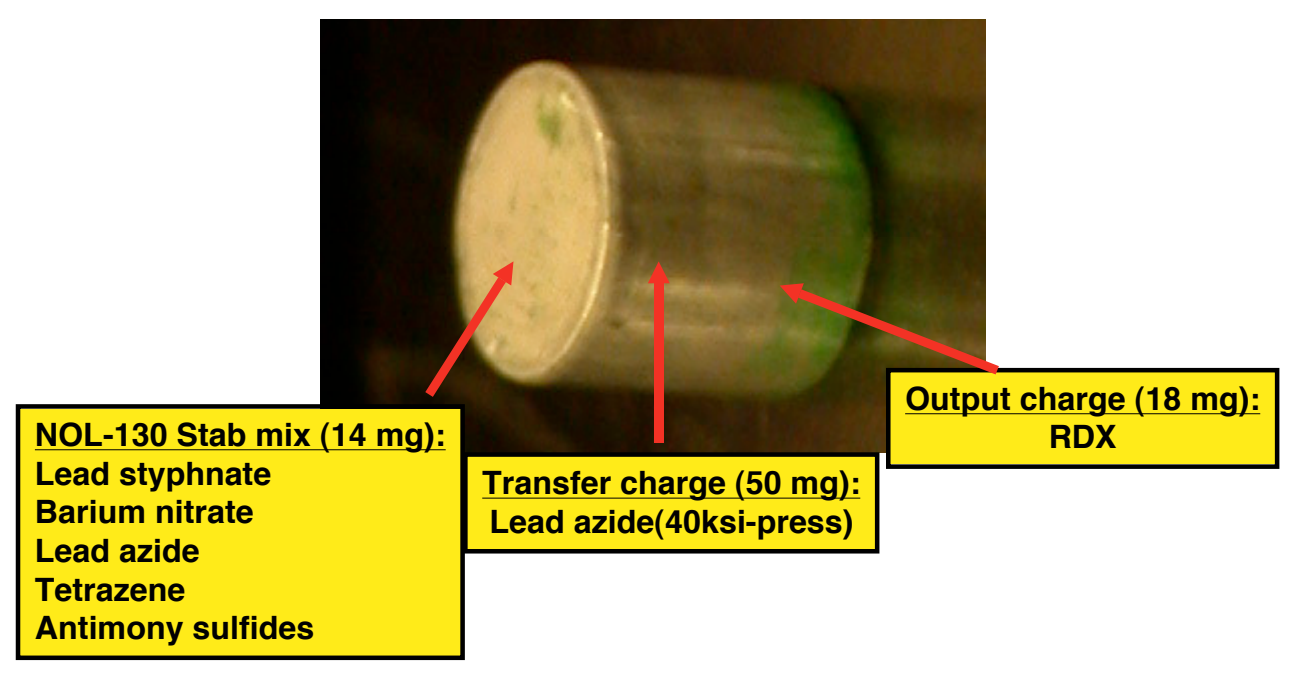

Figure 9. This figure is a photo of a M55 stab detonator that displays the composition and spatial arrangement of the detonation train. The detonator pin fires into the left hand side of the cup with a left to right direction.

Comparison of Figures 8 and 9 show the similarities between current IID testing and nanolaminate-based replacement ones. The pins and cups used are identical. We are using pressed talc powder to mimic the pressed energetic materials that will be in a live M55 with nanolaminate in it. Inert surrogate material (talc) was used for initial testing because lead azide was inappropriate at the time. From the LLNL drop test results we knew the nanolaminate materials could ignite at or above 12 in./oz. At these stab energy input levels lead azide itself will detonate. Therefore it would be impossible to determine if the nanolaminate initiated the lead azide or the alternative. IN addition the LLNL drop test utilizes a steel anvil substrate whereas the ARDEC-TACOM testing utilizes pressed powder as the substrate. The mechanics of each systems are different. In the future the LLNL drop testing must be altered to more closely resemble the ARDEC-TACOM dropweight testing. Therefore, testing done in October 2004 was intended to get a qualitative estimation of the impact energy needed to initiate the nanolaminates when a pressed powder substrate was used.

Both current M55 stab detonators and those with nanolaminates integrated into them were tested at ARDEC-TACOM using their drop-weight test. In this test a one ounce ball is dropped remotely onto a firing pin in contact with the stab detonator. 
Qualitatively detonation is gauged visually through a window in the chamber door. Quantitatively the energy output from the M55 detonator can be determined by measuring the dent profile in a lead, steel, or aluminum plate that was placed in contact with the RDX-containing end of the detonator. The current M55 stab detonator performs at an energy input level of level of $3 \mathrm{in} . / \mathrm{oz}$. The drop-weight test apparatus at Picatinny is shown in Figure 10.

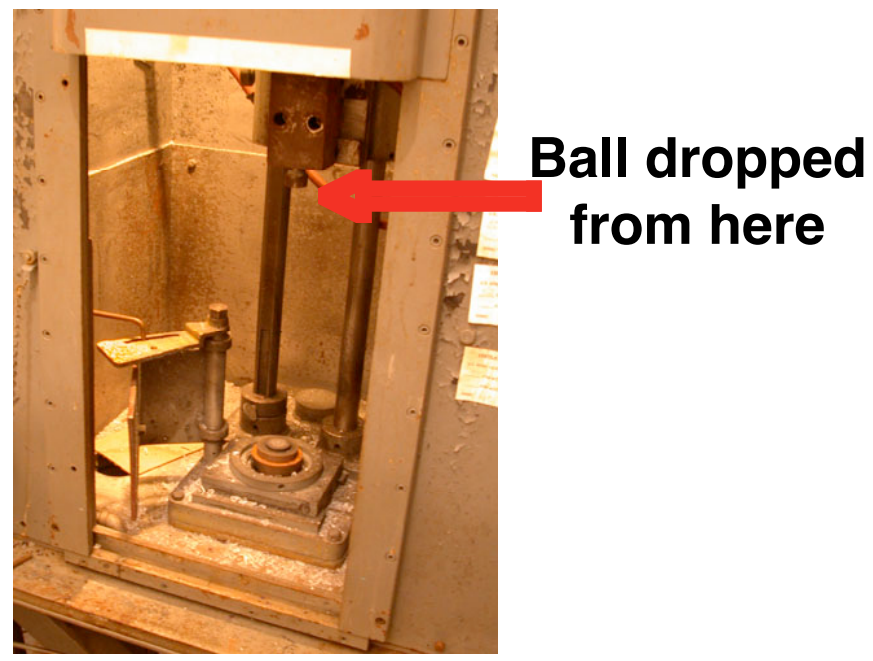

Figure 10. Drop-weight test apparatus at ARDEC-TACOM, Picatinny, NJ

Samples of four different energetic multilayer materials (two different Al/Monel and two different $\mathrm{Al} / \mathrm{Ni}(\mathrm{V})$ materials) were tested at ARDEC-TACOM. A qualitative 'go' or 'no-go' result was determined by visual observation of the impact through the window in the chamber. Since the reacting foils glow brilliantly it is reasonable to assume that their reaction may be observed. However, pierced nanolaminates were removed from fired M55 cups and visually inspected for evidence of reaction. To date, this testing has not indicated that any of the nanolaminate foil materials react to stab initiation in this experimental set up. The M55 pin has sufficient energy to pierce the nanolaminate as is clearly seen in the photo below in Figure 11. 


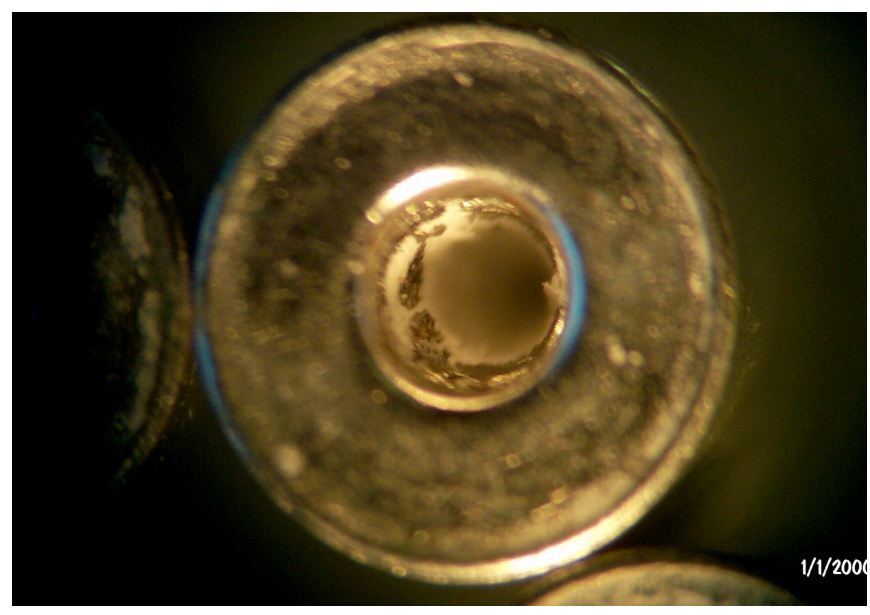

Figure 11. Photo of pierced M55 detonator cup that was loaded with nanolaminate foil and talc.

The M55 detonator shown in Figure 11 is typical for this experiment. As one can see the pin has pierced the cup as well and the nanolaminate. This is clear from the pressed white talc powder seen. All of the foils were tested at a maximum drop height of 10 in./oz. with the same results. We plan on increasing the drop height in future experiments to determine the sensitivity of the nanolaminates when a pressed powder is used as the substrate.

Attempts to physically sensitize the nanolaminates. Energetic materials are commonly enhanced with the addition of chemical or physical sensitizers. Examples of physical sensitizers are powders that have high hardness and high melting points. Typically the best results are obtained with materials with melting points above $550^{\circ} \mathrm{C}$ and fairly coarse $(50-200 \square \mathrm{m})$ particle sizes.[16,17] It is highly possible that physical sensitization of these energetic multilayers is possible. To that end some basic experiments were performed.

Limited experiments were run using several foils stacked upon one another. The thought here was to induce additional frictional forces as one foil slid across another as the pin indented and pierced them. We observed no reaction in the foils using this approach.

In selected experiments sand particles were added to induce hot spot formation heating by friction between two surfaces or a stress risers that induce local shear flow of the nanolaminate. These very limited attempts were not successful. However, the particle size of the sand used was large (1-2mm) compared to that used published studies on sensitization of energetics with inert particles. We feel that this approach is sound and 
intend to vigorously follow it up in the upcoming months using a variety of different grit materials (e.g., alumina, sand, SiC, and ground glass) with varying particle sizes.

Some attempts were made to enhance the sensitivity of the device by altering the pin geometry. A M55 detonator pin is shown below in Figure 12.

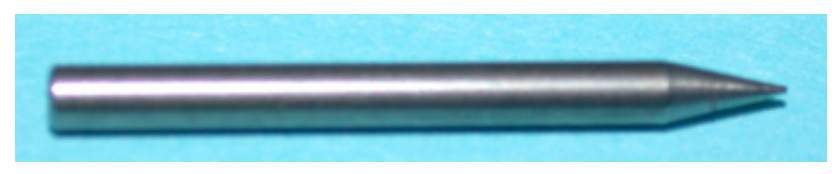

Figure 12. Photo of M55 stab detonator pin, the diameter of the pin head is $230 \square \mathrm{m}$.

A few selected pins were altered and used in experiments. Altering consisted of flattening the head and roughening the conical sides of the firing pin. The alteration of the pin did not appear to sensitize the new devices.

More careful thought upon our observations of the initiation of nanolaminates by mechanical means suggests that a pinching action between two surfaces may be the mode of initiation. In Figure 13 below that pinching action is supplied between the steel substrate and the steel punch.

a)

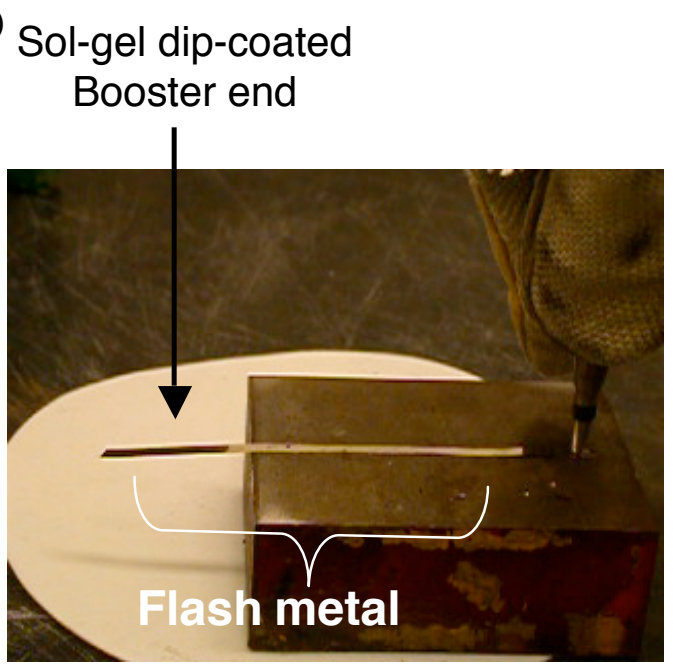

b)

\section{Sol-gel booster}

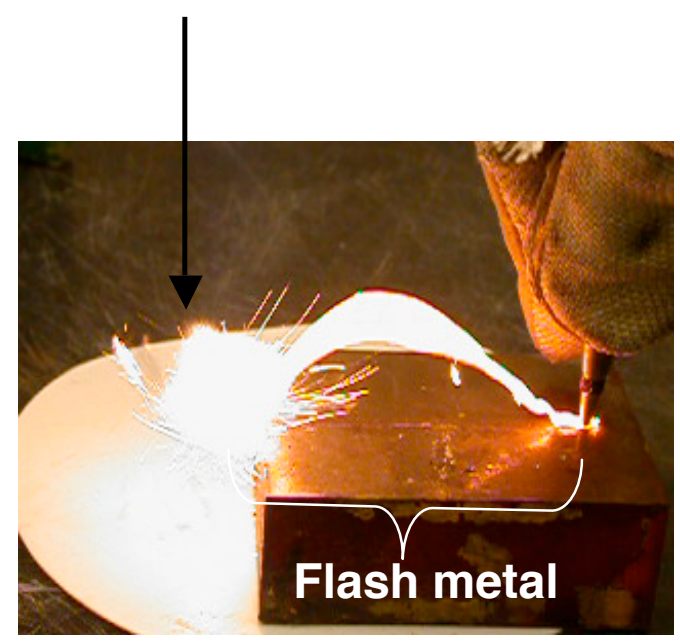

Figure 13. a) Sol-gel $\mathrm{Fe}_{2} \mathrm{O}_{3} / \mathrm{Al}$ coated $\mathrm{Ni} / \mathrm{Al}$ multilayer nanocomposite (the coated portion of the multilayer is on the left end of the foil and b) mechanical initiation of the nanocomposite, using a spring-loaded punch, results in ignition of the energetic sol-gel coating. 
As can be seen from the images, the mechanical pinching stimulus induces the exothermic transformation of the multilayer to its respective intermetallic alloy. The arching glowing foil indicates that the transformation propagates along the foil perpendicular to the punch and migrates to the energetic sol-gel-coated region of the foil. This reaction has sufficient output energy to ignite the more energy dense sol-gel thermite reaction.[12] It is our feeling that attempting to create a pinching mechanism in the M55 detonator when the pin is fired is a suitable way to address the sensitivity. This will be further addressed in the Summary/Future works portion of this report.

New Sol-gel synthesis of nanometric tungsten (VI) oxide $\left(\mathrm{WO}_{3}\right)$. One of the most significant developments in the last year was the successful sol-gel synthesis and characterization of nanostructured tungsten (VI) oxide $\left(\mathrm{WO}_{3}\right)$. Previously, our basic science efforts had focused on sol-gel $\mathrm{Fe}_{2} \mathrm{O}_{3}$. Much has been learned about the synthesis purification and characterization of this material and its energetic composites. Tungsten trioxide is of interest for several important reasons. One, its physical density is fairly high for an oxide $\left(7.2 \mathrm{~g} / \mathrm{cc}\right.$ as compared to $5.2 \mathrm{~g} / \mathrm{cc}$ for $\left.\mathrm{Fe}_{2} \mathrm{O}_{3}\right)$. Literature reports that indicate $\mathrm{Al} / \mathrm{WO}_{3}$ mixes burn more rapidly and explosively than any others. This may be attributed to the high vapor pressure of $\mathrm{WO}_{3}$ products at relatively low temperatures ( $\mathrm{WO}_{3}$ begins to sublime at $<750^{\circ} \mathrm{C}$ ). It has been suggested that gaseous $\mathrm{WO}_{3}$ participates and enhances the combustion of the mixtures containing $\mathrm{WO}_{3}$. This is significant as many thermite and intermetallic reactions are considered gas-less.[18] These gaseous species provide a pressure jump in thermite reactions containing $\mathrm{WO}_{3}$. [19] This is particular relevant in primers and stab detonators as good heat transfer is needed between energetic components and factors such pressure pulse and hot particle ejection contribute to this.

Sol-gel derived $\mathrm{WO}_{3}$ was prepared via two different routes in our laboratory. One method utilizes the epoxide addition method we have reported previously. In summary, the addition of organic epoxides to solutions of transition metal salts under the correct conditions results in the formation of a robust rigid gel made up of particles whose primary dimensions are on the nanometric scale. In these systems the epoxide acts as a proton scavenger by reacted irreversibly with protons from hydrated metal complexes and nucleophiles present in solution. This acts to slowly and uniformly raise the $\mathrm{pH}$ of the solution and promotes the polycondensation of aqua and aqua/hydroxy complexes of the metal through the processes of olation and oxolation. For $\mathrm{WO}_{3}$ gels we have found that the starting material can be either $\mathrm{WOCl}_{4}$ or $\mathrm{WCl}_{6}$. These compounds are inorganic salts of tungsten (VI) that are soluble in ethanol. After adding a small amount of water any of several different organic epoxides can be added to induce gelation. Rigid yellow gels of $\mathrm{WO}_{3}$ can be formed as is shown in Figure 14. 


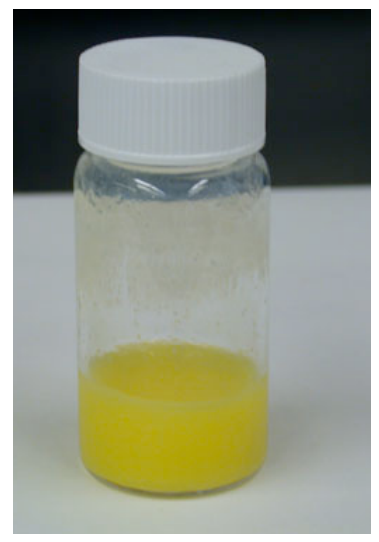

Figure 14. Sol-gel derived $\mathrm{WO}_{3}$ gel.

Suspensions of these gels with Al have been coated onto the surface of nanolaminates using dip or spin coating techniques (see Figure 15 below). Recently the oxidizer bismuth (III) oxide, $\mathrm{Bi}_{2} \mathrm{O}_{3}$, has shown a great deal of promise in thermite systems where gas generation is needed. Presently we have another program investigating the sol-gel synthesis of nano-sized $\mathrm{Bi}_{2} \mathrm{O}_{3}$. Benefits from that program may be realized and utilized here in the coming year.
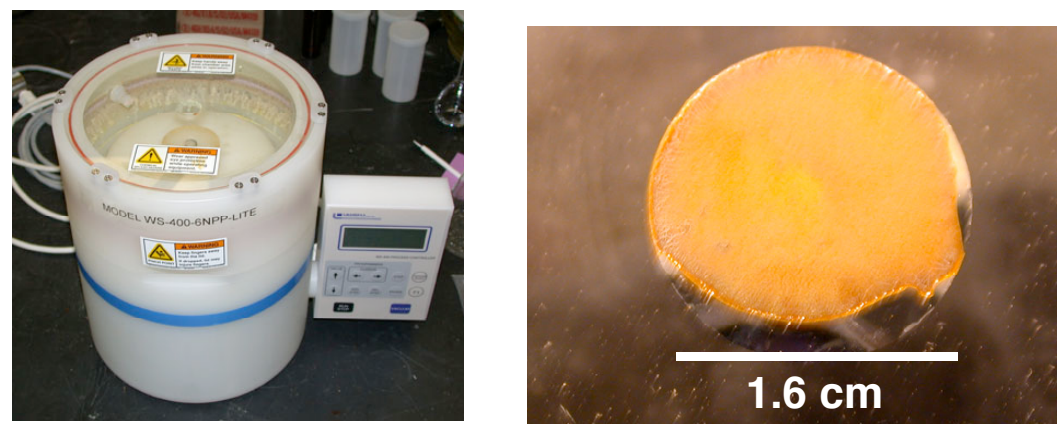

Figure 15. Spin coater and nanolaminate spun coated with sol-gel $\mathrm{Fe}_{2} \mathrm{O}_{3} / \mathrm{Al}$ energetic composite.

We have successfully dip-coated $\mathrm{Ni} / \mathrm{Al}$ flash metal multilayer foil with pyrotechnic-based sol-gel materials. Preliminary experiments have indicated that the conversion of a Ni/Al multilayer to its respective intermetallic generates sufficient energy to ignite the more energetic $\mathrm{Fe}_{2} \mathrm{O}_{3} / \mathrm{Al}$ composite coating. We have successfully used both ultra-fine-grained 
(UFG) nanometer-sized ( 30-100nm diameter) $\mathrm{Al}$ and conventional micron-sized $\mathrm{Al}$ in these types of nanocomposites. It is important to emphasize that these materials can be made successfully using micron-sized Al. Micron-sized Al has been demonstrated to be an effective and reliable component of energetic materials. Although less reactive than the MIC Al, the micron Al has a greater energy density, is safer to work with, and has better aging properties all of which result in more reproducible performance in application.

\section{Summary/Future Work}

Progress on several fronts was made this year. It was demonstrated that reacting nanolaminate has sufficient energy output to initiate the current lead azide transfer charge material. Current and future work in this area is focused on demonstrating this in a stab detonator configuration. This year we demonstrated quantitatively that the sensitivity of the energetic nanolaminates to mechanical stimulus can be engineered by tailoring the microstructural properties of the material. This is very significant as the main obstacle to overcome in this project is the sensitization of the nanolaminates. To be used as replacements in current stab detonators they must be more sensitive to stab impact. Our understanding of the critical parameters that affect sensitivity of the energetic foils allows us to continue to increase their sensitivity through structural manipulation. We intend on vigorously pursuing this in the next year. In addition we believe that physical sensitization of the films may be possible with the use of hard high melting point powders and intend on investigating this. We feel that a pinching mechanism may be

responsible for the initiation of the energetic nanolaminates. With this in mind we intend to try using a small anvil or wire grid integrated into the stab detonator to examine its effect on the efficacy of current energetic nanolaminates to initiate stab detonators.

\section{References}

1. Cooper, P.W. Explosives Engineering, Wiley-VCH, New York. 1996, p.323-326.

2. Mann, A.B.; Gavens, M.E.; Reiss, M.E.; Van Heerden, D.; Bao, G.; Weihs, T.P. J. Appl. Phys. 1997, 82(3), 1178.

3. Gavens, A.J.; Van Heerden, D.; Mann, A. B. ; Reiss, M.E.; Weihs, T.P. J. Appl. Phys., 2000, 87(3), 1255.

4. Barbee, T.W., Jr.; Weihs, T.P. 1996, U.S. Patent 5,547,715.

5. Barbee, T.W., Jr.; Weihs, T.P. 1996, U.S. Patent 5,538,795.

6. Simpson, R.L.; Tillotson, T.M.; Satcher, J.H., Jr.; Hrubesh, L.W.; Gash, A.E. Int. Annu. Conf. ICT (31 $1^{\text {st }}$ Energetic Materials), Karlsruhe, Germany, June 27-30, 2000. 
7. Gash, A.E.; Simpson, R. L.; Tillotson, T.M.; Satcher, J.H., Jr.; Hrubesh, L.W. Proc. 27 ${ }^{\text {th }}$ Int. Pyrotech. Semin. Grand Junction, CO, July 15-21, 2000 p.41-53.

8. Tillotson, T.M.; Gash A.E.; Simpson, R.L.; Hrubesh, L.W.; Thomas, I.M.; Poco, J.F. J. Non-Cryst. Solids 2001, 285, 338-345.

9. Tillotson, T.M.; Hrubesh, L.W.; Simpson, R.L.; Gash. A.E., Patent application \#S.N. 09/586,426

10. Gash, A.E.; Tillotson, T.M.; Satcher, J. H., Jr.; Hrubesh, L. W.; Simpson, R. L. Chem. Mater. 2001, 13, 999.

11. Gash, A.E.; Tillotson, T.M.; Poco, J. F.; Satcher, J. H., Jr.; Hrubesh, L. W.; Simpson, R. L. J. Non-Cryst. Solids 2001, 285, 22-28.

12. Fisher, S.H.; Grubelich, M.C. Proc. $24^{\text {th }}$ Inter. Pyrotech. Sem. 1998, 231.

13. Barbee, T.W. Gash, A.E.; Satcher, J.H.; Simpson, R.L. Proceedings from the $34^{\text {th }}$ ICT Meeting, Karlsruhe, FGR, June 24-28. 31-1.

14. Simpson, L.R.; Foltz, M.F. Report: LLNL Small-Scale Drop-Hammer Impact Sensitivity Test, UCRL-ID-119665, 1995.

15. Chaudhri, M.M. Nature, 1976, 263, 121.

16. Bowden, F.P.; Gurton, O.A. Proc. Royal Soc. 1949, 198.

17. Feld, J.E. Acc. Chem. Res. 1992, 25, 489.

18. Wang L.L.; Munir, Z.A.; Maximov Y.M. J. Mater. Sci.. 1993, 28, 3693.

19. Perry et al. Propellants Explosives and Pyrotechnics, 2004, 29(2), 99. 